\title{
Secondary bronchial botryomycosis due to foreign body aspiration
}

\author{
J. Müller-Quernheim1,3, E. Vollmer2, J. Galle2
}

ABSTRACT: Secondary bronchial botryomycosis due to foreign body aspiration. J. Müller-Quernheim, E. Vollmer, J. Galle.

Botryomycosis is recognised mainly as a visceral disorder with rare cases of pulmonary manifestation. The most frequent cause of pulmonary Botryomycosis is aspiration of a foreign body which induces bacteria to group together instead of spreading out forming conglomerates resembling the granules of Actinomyces. Here we report on the clinical and pathologic findings of a 38-year-old patient without any further predisposing factors. It should be mentioned that the disease was cured following the extraction of a foreign body without the need for any surgery or antibiotic therapy. Factors influencing the course of the disease are discussed below.

Monaldi Arch Chest Dis 2007; 67: 2, 119-121.

1 Department of Pneumology, University Medical Center, Albert-Ludwigs-Universität Freiburg,

2 Division of Clinical and Experimental Pathology, Medical Hospital, Research Center Borstel, Germany.

Correspondence: Dr. Prof. Joachim Müller-Quernheim, Head, Department of Pneumology, University Medical Center, AlbertLudwigs-Universität Freiburg, Killianstr. 5, 79106 Freiburg, Germany; e-mail: joachim.mueller-quernheim@uniklinikfreiburg.de

Botryomycosis is an uncommon bacterial disease caused by a wide spectrum of gram positive and gram negative bacteria that mimics infection by Actinomyces species. A wide spectrum of risk factors defines susceptibility for Botryomycosis. These include aberrant immune reaction, cystic fibrosis, HIV-infection, chronic granulomatous disease, diabetes mellitus, tracheopathia osteochondroplastica, heavy smoking, and foreign body aspiration $[1,2]$. Some cases have been addressed as primary pulmonary or bronchial Botryomycosis since an underlying risk factor could not be found [1]. However, there are cases of primary Botryomycosis in which a risk factor could be identified later in the course of the disease [3]. In the cases of primary and secondary Botryomycosis of the lung reported in the literature, surgical or antibiotic therapy was necessary to reach resolution. However, it should be noted that depending on the underlying disease, progressive and fatal courses may take place.

Here we report a case of bronchial Botryomycosis due to foreign body aspiration which came to resolution without antibiotic treatment following extraction of the foreign body during a diagnostic fiberoptic bronchoscopy.

\section{Case Report}

A 38-year-old woman was admitted for a diagnostic bronchoscopy of a middle lobe syndrome. The patient's history was unremarkable. She was first brought to medical attention four months pri- or to admission when she began experiencing fever and right-side chest pains. Pneumonia was diagnosed and macrolide therapy initiated.

Symptomatic improvement was observed, however, sub-febrile temperatures and chest discomfort persisted. Laboratory investigation revealed a modest increase of $\mathrm{C}$-reactive protein without any other remarkable finding which might have guided diagnostic procedures. Thus, she was treated by her general practitioner with several courses of second generation cephalosporines but blunt symptoms maintained. Eventually, a chest$\mathrm{X}$-ray was taken which revealed an atelectasis of the right middle lobe and the patient was admitted for bronchoscopy.

Upon admission she was unwell, without temperature or any remarkable finding in the laboratory investigation. A bronchoscopic examination revealed a whitish exophytic material (figure 1) which completely occluded the ostium of the middle lobe. Biopsy forceps were protruded in the middle lobe ostium between the bronchial wall and the exophytic mass to take a biopsy from its base. Retraction of the forceps mobilised the exophytic mass entirely. Since it could not pass through the instrument channel of the bronchoscope the instrument was withdrawn with the forceps holding the exophytic mass at its tip. During the passage through the nose the exophytic mass was lost and could not be recovered. However, the material retained in the forceps was sent for histological examination. The bronchoscope was introduced a second time and a free lumen of the middle lobe ostium with inflammatory mucosal reaction could 


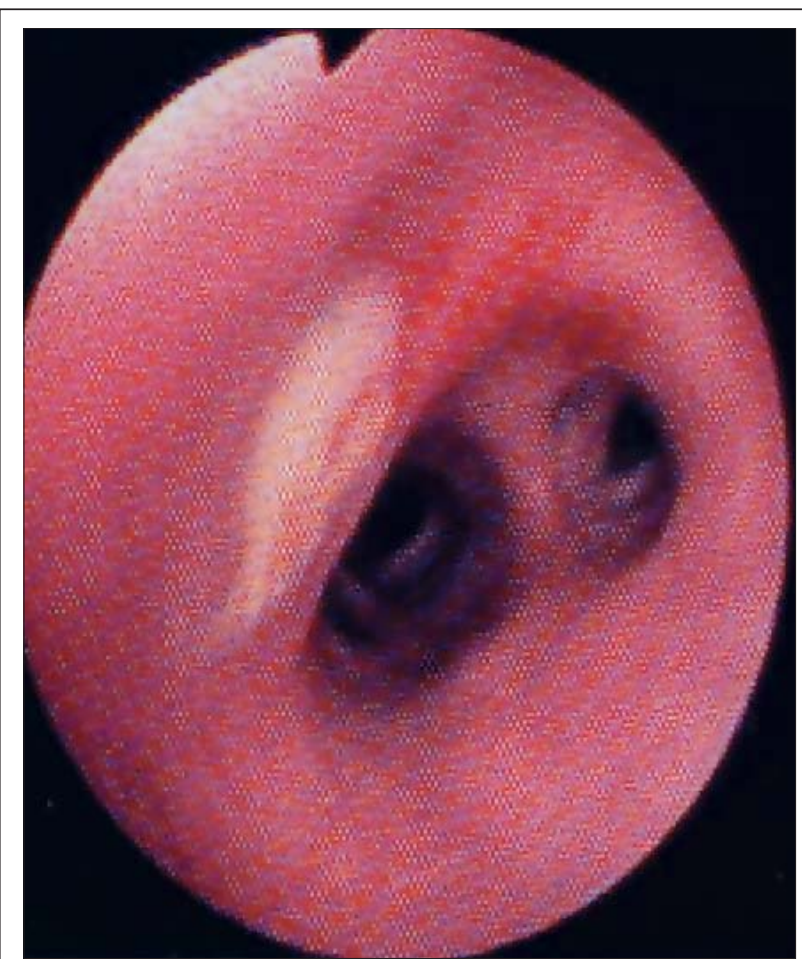

Fig. 1. - Exophytic material in the ostium of the middle lobe.

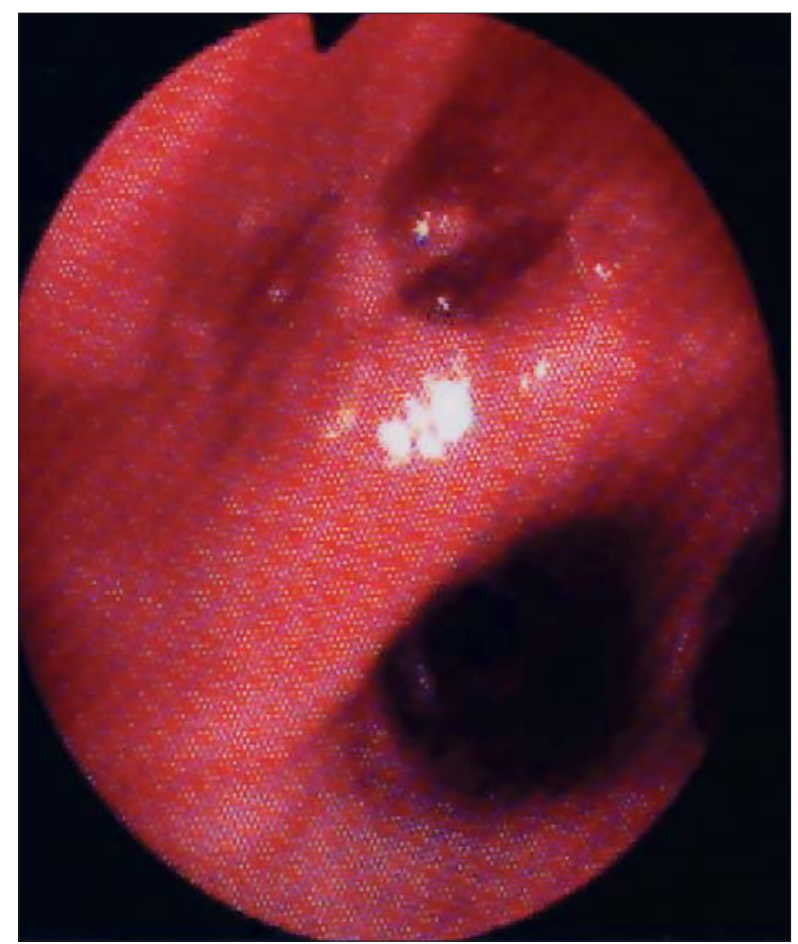

Fig. 2. - Inflammatory reaction of the middle lobe ostium after extirpation of the foreign body.

be observed (figure 2). From its rim a mucosal biopsy was taken.

A histological examination revealed that no human tissue, only debris was present in the biopsy of the exophytic mass. The biopsy of the bronchial mucosa of the middle lobe ostium did not contain any neoplastic cells but sulfuric granule-like material surrounded by a necrotic substance and inflammatory cells on haematoxylin and eosin staining (figure 3 ). The granules com- prise densely packed bacteria surrounded by proteinaceous material. This focus is surrounded by chronic inflammatory cells composed by polymorph nuclear leukocytes, monocytes, and foreign body giant cells within a matrix of fibrous tissue (figure 4). This histological pattern is referred to as the Splendore-Hoeppli phenomenon $[4,5]$. This phenomenon is observed in Actinomyces, Botryomycosis and other infections. The diseases are satisfactorily distinguished when additional staining such as PAS-, and Gomori-Grocott-staining fail to identify branching organisms or acid-fast bacilli as was the case with our patient.

After the bronchoscopy which resulted in the diagnosis of Botryomycosis, the patient improved without any further therapy and all her symptoms resolved. After the diagnosis was explained to the patient she was reminded by her children and recalled an event of aspiration of a broccoli during a family dinner about five months prior to the development of symptoms which led to the first antibiotic therapy. She most saw her practitioner eight months later for the last time for a follow-up where she presented without any symptoms and a normal chest-X-ray was taken.

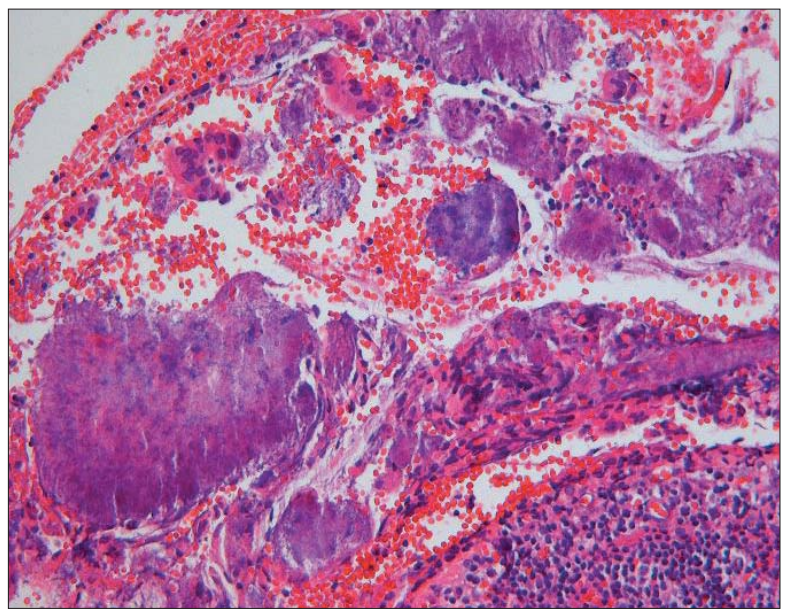

Fig. 3. - Bacterial grain in cellular debris from the rim of the middle lobe ostium. Haematoxylin-eosin stain. Original magnification 100-fold.

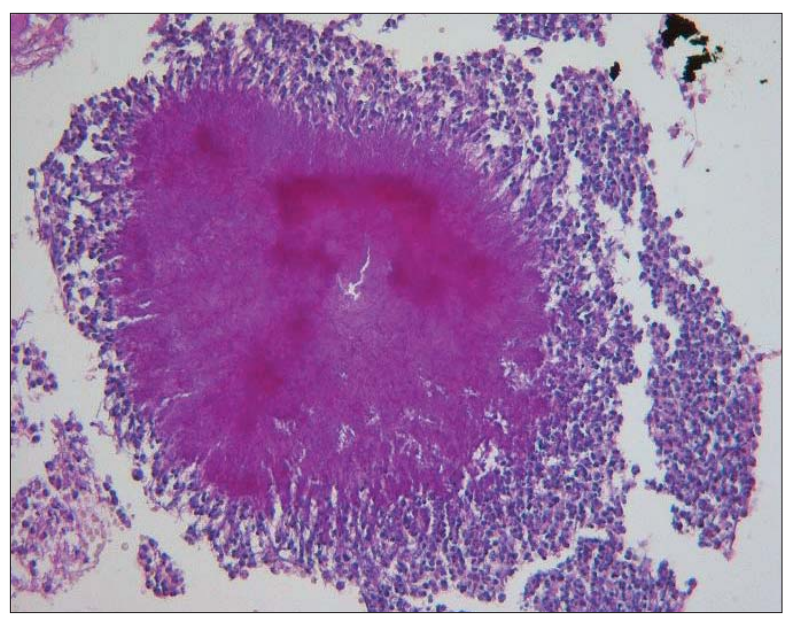

Fig. 4. - Splendore-Hoeppli phenomenon. At the edge of a cluster of bacteria is radiating eosinophilic material. Haematoxylin-eosin stain. Original magnification 150-fold. 


\section{Discussion}

Botryomycosis has been called the "champignon de castration" because of the original descriptions of a disease after castration in horses. It was described as early as 1870 by Bollinger [6]. Howev$\mathrm{er}$, it was a misnomer as the disease was initially thought to be fungal. Fourteen years later the term Botryomycosis was coined by Rivolta, referring to the grapelike appearance of the granules (botry) and the presumed fungal etiology (mycosis) [7]. The most characteristic feature of the disease is that the bacteria, instead of spreading throughout the infected tissue, group together to form conglomerates resembling the sulfuric granules of Actinomyces.

Infection with Actinomyces species usually requires disruption of a mucosal barrier. This disruption may have a iatrogenic cause, as in surgery, or by a foreign body such as an intrauterine device [8, 9]. Pulmonary Actinomycosis, however, is usually attributed to aspiration. The factors that induce the formation of the granules of Botryomycosis have not been identified, but both bacterial and host factors have been implicated.

The pathogenesis of Botryomycosis has not yet been determined. The following factors may be responsible for its pathogenesis: local trauma, foreign body, size of bacteria inoculum, organisms of low virulence; and immunodeficiency of host.

These factors appear to lead to a state of equilibrium between the host and the infecting agent. Histopathologically, this leads to granulation tissue, newly formed capillaries, epithelial cells, giant cells and micro-abscesses composed of neutrophils as well as the typical granules [5].

Predisposing risk factors have been reported in most of the cases of human bronchial or pulmonary Botryomycosis [1, 10]. Visceral Botryomycosis, also well recognised, is relatively rare with few reports of mostly fatal cases in the literature $[1,11]$. Those who survived had surgically resectable lesions.

The literature suggests that the presence of a foreign body is required to provoke the granule formation in bronchial and pulmonary Botryomycosis. However, our patient could not recall having aspirated a broccoli in the past which protracted diagnosis. The size of the inoculum, virulence of the organism, and host defenses are believed to be co-factors in the bacterial formation of granules. There is also evidence to show that immunosuppression was an important cofactor. No risk factors, such as diabetes mellitus, HIV-infection, or cystic fibrosis, were present in our patient.
Moreover, there were no risk factors for aspiration such as neuromuscolar disease or any addiction. A delay of more than five years of a diagnosis of bronchial Botryomycosis has been reported. During those long dwelling times extensive Botryomycosis requesting surgical resection can develop. In our case it was due to aspiration of vegetable and mimicked bronchial carcinoma by a consolidation in the lower lobe with a cavity and mucopurulent secretion. After the diagnosis had been made antibiotic therapy was sufficient to induce complete resolution of the disease [3]. In the case presented the absence of any further predisposing factor and the only short dwelling time of the foreign body in the ostium of the middle lobe might be the reasons for complete and quick resolution of disease without any further antibiotic treatment just after the removal of the foreign body. In conclusion, the early referral for a diagnostic bronchoscopy is most likely the reason for the fortunate outcome and of avoiding serious organ damage.

\section{References}

1. Bersoff-Matcha SJ, Roper CC, Liapis H, Little JR. Primary pulmonary botryomycosis: case report and review. Clin Infect Dis 1998; 26: 620-4.

2. Shih JY, Hsueh PR, Chang YL, et al. Tracheal botryomycosis in a patient with tracheopathia osteochondroplastica. Thorax. 1998; 53: 73-5.

3. Tuggey JM, Hosker HS, DaCosta P. Primary pulmonary botryomycosis: a late complication of foreign body aspiration. Thorax 2000; 55: 1068-9.

4. Dail D, Hammer S. Pulmonary Pathology. New York: Springer Verlag; 1988. p. 160-2.

5. Travis W, Colby T, Koss M, Rosado-de-Christenson M, Müller N, King Jr T. Non-neoplastic disorders of the lower respiratory tract. Washington, DC: American Registry of Pathology and the Armed Forces Institute of Pathology; 2002. p. 573-4, 621.

6. Bollinger O, Modlich F. Mycosis der Lunge beim Pferde. Virchow's Arch Pathol Anat 1870; 49: 583-6.

7. Rivolta S. Del micelio e delle varieta e specie de discomiceti patogeni. Giorn di anat fisiol e patol d animali 1884; 16: 181-98.

8. Putman HC Jr, Dockerty MB, Waugh JM. Abdominal actinomycosis; an analysis of 122 cases. Surgery 1950; 28: 781-800.

9. Henderson SR. Pelvic actinomycosis associated with an intrauterine device. Obstet Gynecol 1973; 41: 726-32.

10. Katapadi K, Pujol F, Vuletin JC, Katapadi M, Pachter BR. Pulmonary botryomycosis in a patient with AIDS. Chest 1996; 109: 276-8.

11. Multz AS, Cohen R, Azeuta V. Bacterial pseudomycosis: a rare cause of haemoptysis. Eur Respir J 1994; 7 : 1712-3. 